\title{
ICTIOFAUNA DE UN SISTEMA FLUVIAL RECEPTOR DE AGUAS SERVIDAS: RESPUESTAS A UNA NUEVA PLANTA DE TRATAMIENTO (RIO QUILQUE, CHILE CENTRAL)
}

\author{
ICHTHYOFAUNA OF A FLUVIAL SYSTEM RECEPTOR OF URBAN \\ WASTEWATER: RESPONSES TO A NEW TREATMENT PLANT (QUILQUE \\ STREAM, CENTRAL CHILE)
}

\author{
Evelyn Habit, Oscar Parra \& Claudio Valdovinos \\ Unidad de Sistemas Acuáticos, Centro de Ciencias Ambientales EULA-Chile, Universidad de Concepción, Chile. \\ Casilla 160-C, Concepción, Chile. ehabit@udec.cl
}

\begin{abstract}
RESUMEN
El sistema fluvial Quilque (orden 3) recibe las descargas de aguas servidas de una población aproximada de 115.000 habitantes y de 19 industrias. Estos residuos se vertían sin tratar directamente al curso del río hasta fines del año 2002, cuando se puso en marcha una planta de tratamiento de aguas servidas. Para analizar los efectos de estas descargas sobre la fauna de peces, se estudió la calidad del agua y estructura comunitaria de la ictiofauna a lo largo de ocho estaciones ubicadas desde aguas arriba de los vertidos hasta $300 \mathrm{~m}$ aguas abajo del efluente de la planta de tratamiento. Tanto la calidad del agua como la fauna de peces mostraron una leve mejoría luego de cinco meses de operación de la planta de tratamiento. Aun cuando se redujeron las concentraciones de $\mathrm{DBO}_{5}$ sólidos suspendidos, nitrógeno y coliformes fecales, el río conduce una carga contaminante importante desde aguas arriba y mantiene remanentes en los sedimentos del lecho del río. La ictiofauna se encuentra empobrecida, pero evidencia un proceso de recolonización del sistema, desde las partes altas hacia el tramo inferior que recibía las descargas. Sin embargo, aún se mantienen sectores sin peces, debido a las altas concentraciones de $\mathrm{DBO}_{5}$. Las primeras especies en recolonizar aguas abajo son nativas (Trichomycterus areolatus y Percilia irwini).
\end{abstract}

Palabras laves: Ictiofauna, aguas servidas, planta tratamiento, Quilque, Chile.

\section{ABSTRACT}

The Quilque River ( $3^{\text {rd }}$ order) receives urban wastewater discharge from a population of $c a .115,000$ inhabitants and 19 industrial effluents. These residues were discharged without treatment until the end of 2002, when a wastewater treatment plant was constructed. To determine effects of wastewater and industrial effluents on the ichthyofauna, we studied water quality and fish community structure at eight stations distributed from upstream of the areas of the discharge to $300 \mathrm{~m}$ downstream of the effluent of the wastewater treatment plant. Both water quality and the fish community showed a slight improvement five months after the treatment plant began operation. Concentrations of $\mathrm{BOD}_{5}$, suspended solids, nitrogen, and fecal coliforms decreased; however, the river contained a significant pollution load from upstream areas, and remnants in the sediments of the riverbed. The fish fauna is impoverished, but there appears to be evidence of a recolonization from upper reaches towards the lower zone receiving the pollution discharges. Areas without fishes still exist due to high concentrations of $\mathrm{BOD}_{5}$. The first species recolonizing the polluted areas are the native fishes Trichomycterus areolatus and Percilia irwini.

KEYwords: Ichthyofauna, municipal wastewater, treatment plant, Quilque, Chile.

\section{INTRODUCCION}

Las descargas de aguas servidas domésticas sin tratar generan múltiples efectos negativos en los cuerpos de aguas receptores. El impacto primario es el deterioro de la calidad del agua debido al aporte de materia orgánica, nutrientes (fósforo, nitrógeno), demanda bioquímica de oxígeno $\left(\mathrm{DBO}_{5}\right)$, sólidos y 
bacterias coliformes, lo que puede conducir a la eutrofización del sistema acuático (Vollenweider 1968). A su vez los cambios en el hábitat acuático por incremento de la turbidez, tasas de sedimentación y biomasa algal (Scrimgeour \& Chambers 2000), provocan alteraciones en las cadenas tróficas del sistema receptor (Dyer et al. 2003). En las comunidades de peces, el nivel de nutrientes no sólo influye en la producción íctica, sino también en la composición específica de la comunidad (Colby et al. 1972). En sistemas con mayor trofía e inferior calidad del agua se produce la dominancia de especies generalistas y tolerantes, las cuales desplazan a las especies nativas y se pierden los depredadores tope (Ganasan \& Hughes 1998, Parra et al. 2003, Porter \& Janz 2002, Wolter et al. 2000). Por ello, la eutrofización de los cursos de agua es una de las principales amenazas para la conservación de la ictiofauna dulceacuícola (Saunders et al. 2002).

El tratamiento de las aguas servidas domésticas implica la reducción de la carga orgánica y, principalmente, de la carga microbiológica que es vertida directamente a los cuerpos acuáticos. Por esto, es esperable que los sistemas receptores presenten un mejoramiento de sus condiciones ecológicas una vez eliminadas las descargas de los vertidos sin tratar. En Chile, la nueva normativa ambiental (DS 90/00, Minsecpre 2001) ha significado la puesta en marcha de plantas de tratamiento, conllevando a la clausura de los alcantarillados que descargaban directamente a los sistemas acuáticos y a la generación de una única descarga de aguas servidas tratadas. A partir del año 2000 han entrado en operación 17 nuevas plantas de tratamiento en la Región del Biobío, entre las que se cuenta la de la ciudad de Los Angeles (37²8' S, 72²1' O). Esta planta sirve a una población de 114.963 habitantes y a 19 industrias y recibe una carga diaria de 6 Ton $\mathrm{DBO}_{5} /$ día, de la cual el $98 \%$ es de origen doméstico (Alarcón \& Román 2003). Tanto el nuevo efluente de la planta como las anteriores cinco descargas de aguas servidas sin tratar, se vierten al sistema fluvial Quilque. Este corresponde a un río de tercer orden que cruza directamente por la zona urbana de la ciudad de Los Angeles para luego desembocar al río Guaqui, tributario de la zona media del río Bío-Bío. Para determinar los efectos generados por la operación de esta nueva planta de tratamiento sobre el ecosistema fluvial receptor, se efectuó un estudio de la calidad del agua y comunidad íctica en las situaciones antes y después de su puesta en marcha.

\section{Metodología}

El estudio se realizó en un tramo del río Quilque ubicado entre el ingreso a la ciudad de Los Angeles hasta $300 \mathrm{~m}$ aguas abajo de la evacuación del efluente de la planta de tratamiento. Se efectuaron dos muestreos, el primero en la situación sin planta de tratamiento (22 de julio del 2002), y el segundo cinco meses después de la puesta en marcha de la planta de tratamiento (12 de mayo del 2003).

Las estaciones de muestreo se establecieron sobre la base de la ubicación de las descargas de aguas servidas sin tratar y de la planta de tratamiento (Fig. 1), estableciendo dos estaciones de referencia (aguas arriba de los vertidos, es decir, sin influencia directa de efluentes de aguas servidas de la ciudad de Los Angeles) y seis estaciones con influencia de efluentes domésticos. Las estaciones E1 y E2 se ubicaron a $1000 \mathrm{~m}$ y $100 \mathrm{~m}$ aguas arriba de la primera descarga sin tratar, respectivamente. Las estaciones E3 y E4 se ubicaron a 50 y $300 \mathrm{~m}$ aguas abajo de la descarga No3 Sur, respectivamente. Las estaciones E5 y E6 se localizaron a 50 y $400 \mathrm{~m}$ aguas abajo de las descargas $\mathrm{N}^{\circ} 3$ Norte, $\mathrm{N}^{\circ} 4$ y $\mathrm{N}^{\circ} 5$, respectivamente. La estación E7 se ubicó en el punto de descarga de la planta de tratamiento de aguas servidas y la estación E8 a 300 m aguas abajo de dicha descarga. En cada estación se tomaron muestras para análisis de calidad del agua (oxígeno disuelto, DQO, $\mathrm{DBO}_{5}$, sólidos suspendidos totales, fósforo total, nitrógeno Kjeldahl y coliformes fecales), se muestrearon peces y se midió el caudal utilizando un correntómetro Gurley (estaciones 1, 5 y 8). Para el muestreo de agua se siguió lo establecido en el Instructivo Presidencial para la dictación de Normas Secundarias de Calidad Ambiental para Aguas Continentales Superficiales y Aguas Marinas (Conama 2002) y aquellas recomendadas por el Standard Methods (APHA 1998). In situ se midieron las variables temperatura (termómetro de mercurio, graduación de $0,1{ }^{\circ} \mathrm{C}$ de sensibilidad), $\mathrm{pH}$ (Schott Gerate Mod. Handylab 2) y conductividad (Cole Parmer Mod. 19820). Los análisis químicos y microbiológicos fueron realizados en los laboratorios de Química Ambiental y Biología Ambiental del Centro EULA-Chile de la Universidad de Concepción, utilizando la metodología indicada en la Tabla I. 


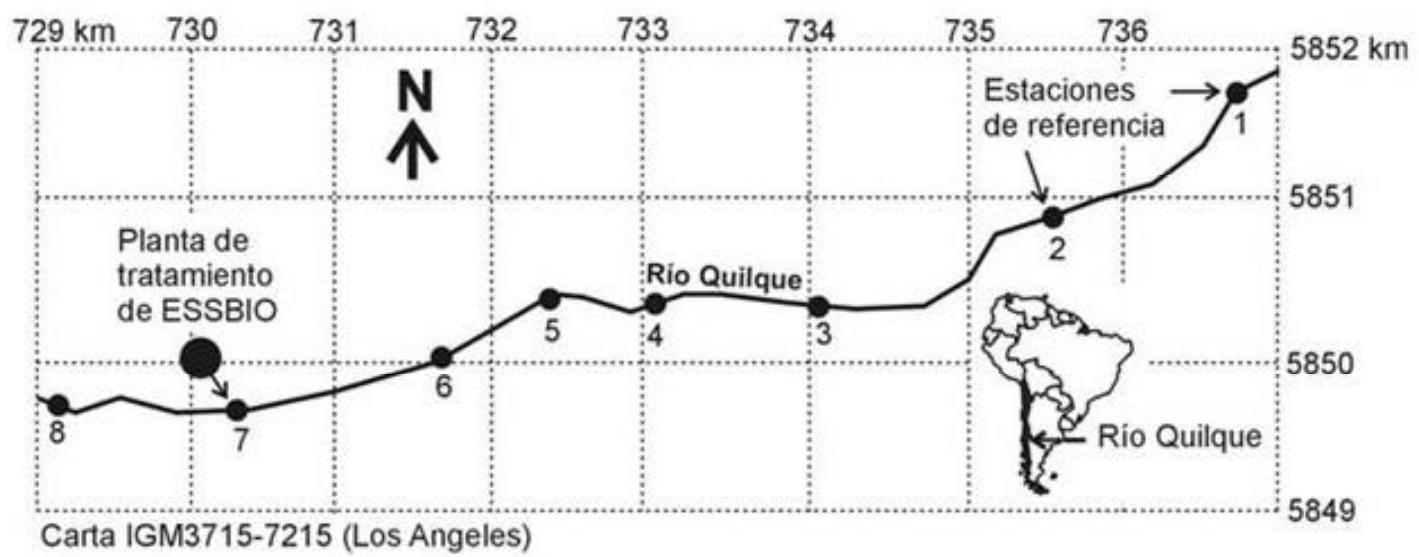

Figura 1. Ubicación de las estaciones de muestreo a lo largo del sistema fluvial Quilque.

FIGURE 1. Localization of the sampling stations along the Quilque fluvial system.

El muestreo de peces se efectuó utilizando como arte de pesca un equipo de pesca eléctrica EFKO. El esfuerzo de captura fue de $50 \mathrm{~m}^{2}$ por 15 minutos de pesca. Los ejemplares capturados fueron determinados taxonómicamente, medidos con un vernier de $0,1 \mathrm{~mm}$ de precisión y pesados en balanza digital de $0,01 \mathrm{~g}$ de precisión. En terreno se analizó su condición externa en cuanto a la presencia de lesiones evidentes y/o ectoparásitos y luego fueron devueltos a su hábitat.

Se estimó la abundancia como captura por unidad de esfuerzo (cpue) y biomasa por unidad de esfuerzo (bpue), además del índice o factor de condición como indicador del "bienestar" de los ejemplares. Las cpue y bpue de ambos muestreos fueron comparados estadísticamente utilizando el test no paramétrico de Mann-Whitney. Para el análisis de la diversidad se determinaron los parámetros comunitarios riqueza específica, diversidad de Shannon $\left(\log _{10}\right)$ y Equidad (Magurran 1988). El análisis de agrupamientos entre las estaciones de muestreo se realizó en base a las capturas por unidad de esfuerzo transformadas mediante $\log (\mathrm{x}+1)$, utilizando el índice de Bray-Curtis (1957) y el método de la media ponderada (Field et al. 1982).

\section{RESULTADOS}

En ambos muestreos se observó una notoria diferencia de la calidad del agua entre las estaciones E1 y E2, con aguas de regular calidad, con respecto a las estaciones E3 a la E8 con aguas de regular a mala calidad (Tabla I). Las principales variables que inciden negativamente en la calidad del agua del río correspondieron a la $\mathrm{DBO}_{5}$, sólidos suspendidos totales y coliformes fecales. En la condición del río con planta de tratamiento se verificó una leve mejoría de la calidad del agua, especialmente en las variables $\mathrm{DBO}_{5}$, sólidos suspendidos totales y coliformes fecales. En el segundo muestreo fue notoria la reducción de coliformes fecales en la estación ubicada directamente aguas abajo de la planta de tratamiento (E8), lo cual se explica por el funcionamiento de la nueva planta, la cual utiliza cloro.

La composición específica, riqueza y distribución de la ictiofauna varió en ambas épocas de muestreo y entre las ocho estaciones. La riqueza específica aumentó en el segundo muestreo tanto en las estaciones de referencia como en aquellas que recibían las descargas sin tratar (Tabla II). 
Ictiofauna del río Quilque: НАBIT, E. ET AL.

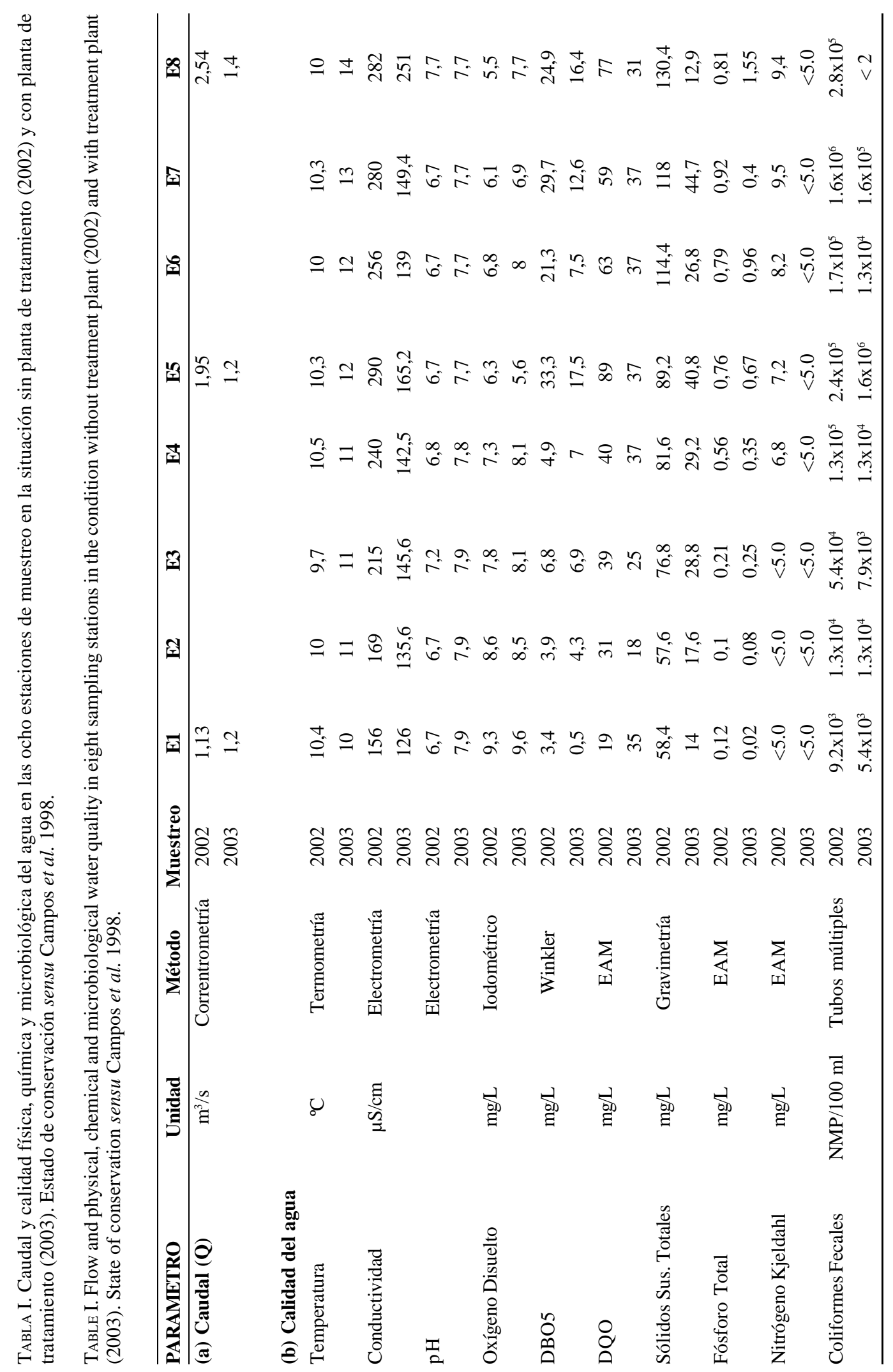


Gayana 69(1), 2005

TABLA II. Composición específica de la fauna íctica y número de individuos capturados en el río Quilque en la situación sin planta de tratamiento (2002) y con planta de tratamiento (2003).

TABLE II. Specific composition of the fish fauna and number of individuals captured in the Quilque stream in the condition without treatment plant (2002) and with treatment plant (2003).

\begin{tabular}{|c|c|c|c|c|c|c|c|c|c|c|c|}
\hline \multirow[t]{2}{*}{ Especie } & Estado conservación & \multicolumn{2}{|l|}{$\mathrm{E} 1$} & \multicolumn{2}{|l|}{ E2 } & E3 & \multicolumn{3}{|c|}{ E4 } & \multicolumn{2}{|l|}{ E5 } \\
\hline & & 2002 & 2003 & 2002 & 2003 & 2002 & 2003 & 20022 & 2003 & 2002 & 2003 \\
\hline $\begin{array}{l}\text { Basilichthys australis } \\
\text { Eigenmann, } 1927\end{array}$ & Vulnerable & 3 & & 1 & & & & & & & \\
\hline $\begin{array}{l}\text { Cheirodon galusdae } \\
\text { Eigenmann, } 1927\end{array}$ & Vulnerable & 3 & & 1 & 13 & & & & & & \\
\hline $\begin{array}{l}\text { Percilia irwini } \\
\text { Eigenmann, } 1927\end{array}$ & En Peligro & 1 & 16 & & 3 & & & & & & 2 \\
\hline $\begin{array}{l}\text { Diplomystes nahuelbutaensis } \\
\text { Arratia, } 1987\end{array}$ & En Peligro & & & & 1 & & & & & & \\
\hline $\begin{array}{l}\text { Trichomycterus areolatus } \\
\text { (Valenciennes, 1848) }\end{array}$ & Vulnerable & & 6 & & 3 & & 6 & & 3 & & \\
\hline $\begin{array}{l}\text { Brachygalaxias bullocki } \\
\text { (Regan, 1908) }\end{array}$ & Vulnerable & & 3 & & & & & & & & \\
\hline $\begin{array}{l}\text { Oncorhynchus mykiss } \\
\text { (Walbaum, 1792) }\end{array}$ & Introducida & & & & & & & & & & \\
\hline & otal Individuos & 7 & 26 & 2 & 20 & $\mathbf{0}$ & 6 & $\mathbf{0}$ & 3 & $\mathbf{0}$ & 2 \\
\hline & Total Especies & 3 & 4 & 2 & 4 & $\mathbf{0}$ & 1 & $\mathbf{0}$ & 1 & $\mathbf{0}$ & 1 \\
\hline
\end{tabular}

Durante el primer muestreo, pre-funcionamiento de la planta de tratamiento, sólo se encontraron peces en las estaciones E1 y E2, ubicadas aguas arriba de las descargas, mientras que en la situación con planta de tratamiento la ictiofauna se distribuyó hasta la estación E5 (Tabla II). Las estaciones E6, E7 y E8 no presentaron peces en ambos muestreos. En la condición sin planta de tratamiento se registraron sólo tres especies nativas, riqueza que aumentó a seis en el segundo muestreo. De éstas, cinco fueron especies nativas y una introducida. De acuerdo a Campos et al. (1998), dos de las especies nativas encontradas se encuentran clasificadas "en peligro" (Percilia irwini y Diplomystes nahuelbutaensis). La primera estuvo presente en ambos muestreos, en tanto que $D$. nahuelbutaensis se capturó sólo en la situación con planta de tratamiento, en la estación E2.

Las especies que mostraron mayor incremento en abundancia y distribución entre ambos muestreos correspondieron a Trichomycterus areolatus y $P$. irwini. Tanto la captura como la biomasa por unidad de esfuerzo presentan un incremento en la situación con planta de tratamiento, pero no estadísticamente significativo (Tabla III MannWhitney para cpue, $\mathrm{Z}=-1,88 ; \mathrm{p}>0,05$ y bpue, $\mathrm{Z}$ $=-1,14 ; \mathrm{p}>0,1)$.

La diversidad de Shannon aumentó en el segundo muestreo en las estaciones E1 y E2, debido a la mayor riqueza específica, pero la equidad disminuyó en ambos casos, debido a la dominancia de $P$. irwini en la estación E1 y de Cheirodon galusdae en E2 (Tabla IV). Las estaciones E3, E4 y E5 presentaron una diversidad de Shannon nula, debido a la presencia de una única especie.

El dendrograma basado en la que muestra dos grupos principales con muy baja similitud entre ellos (9\%). Este agrupamiento queda conformado por un clado correspondiente a estaciones con una ictiofauna empobrecida (E1 y E2 en 2002, y E5 en 2003), y un segundo clado conformado por estaciones que muestran una recuperacion de su fauna de peces (estaciones E1 a E4 en el 2003). Los factores de condición indican que el estado general de las distintas especies en ambas épocas de muestreo fue bueno, con valores característicos para cada especie, sin presencia de lesiones y ectoparásitos (Tabla V). 
Ictiofauna del río Quilque: НАBIT, E. ET AL.

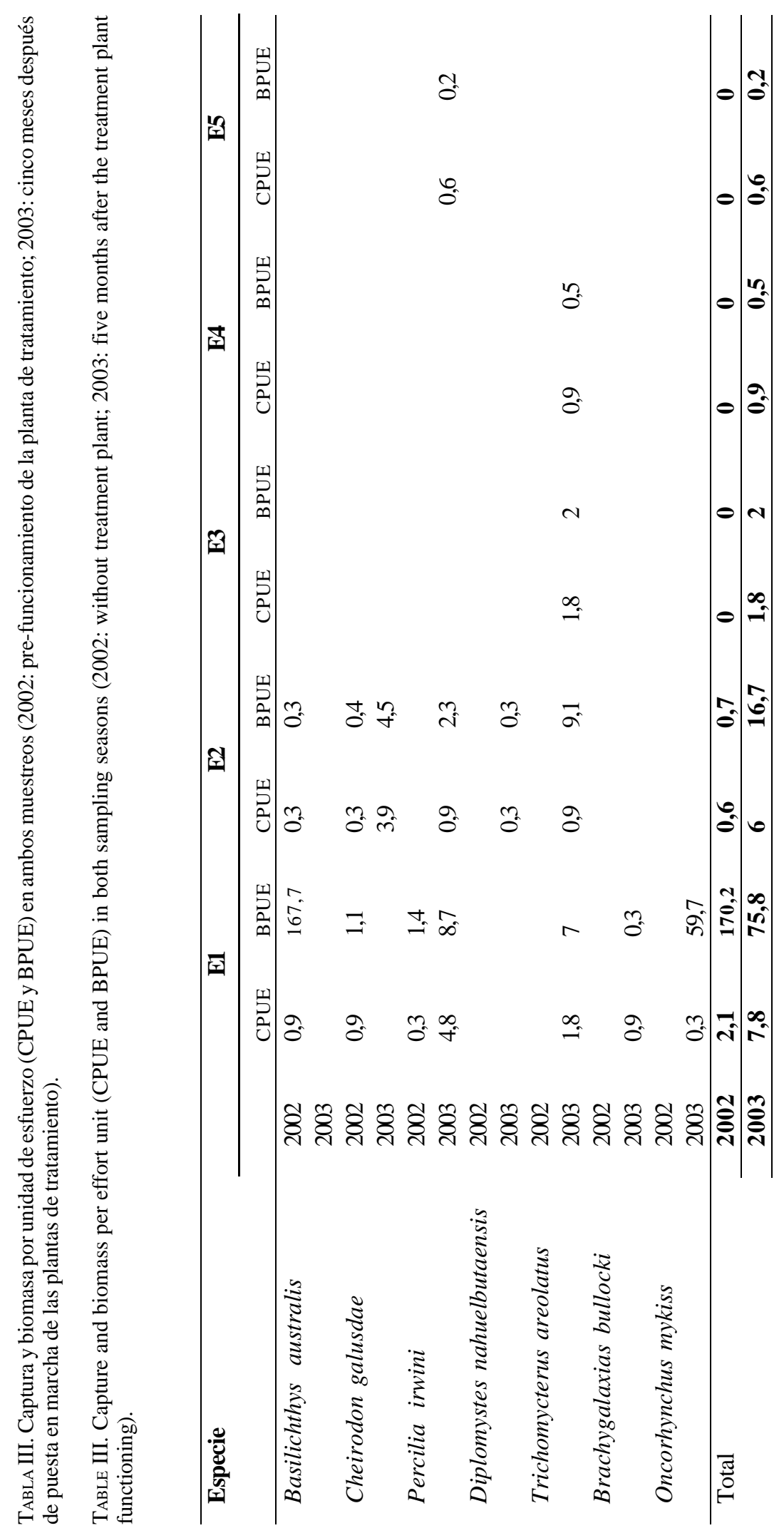


TABLA IV. Riqueza específica, diversidad de Shannon y equidad de la fauna íctica en ambos muestreos (2002: sin planta de tratamiento, 2003: con planta de tratamiento).

TABLE IV. Species richness, Shannon diversity and evenness of the fish fauna in both sampling seasons (2002: without treatment plant, 2003: with treatment plant).

\begin{tabular}{lcccccccccc}
\hline Parámetro & \multicolumn{2}{c}{ E1 } & \multicolumn{2}{c}{ E2 } & \multicolumn{2}{c}{ E3 } & \multicolumn{2}{c}{ E4 } & \multicolumn{2}{c}{ E5 } \\
\cline { 2 - 12 } & 2002 & 2003 & 2002 & 2003 & 2002 & 2003 & 2002 & 2003 & 2002 & 2003 \\
\hline $\begin{array}{l}\text { Riqueza } \\
\text { específica }\end{array}$ & 3 & 4 & 2 & 4 & 0 & 1 & 0 & 1 & 0 & 1 \\
$\begin{array}{l}\text { Diversidad } \\
\text { de Shannon (H') }\end{array}$ & 1,44 & 1,46 & 1,00 & 1,44 & 0 & 0 & 0 & 0 & 0 & 0 \\
Equidad (E) & 0,91 & 0,73 & 1,00 & 0,72 & 0 & 0 & 0 & 0 & 0 & 0 \\
\hline
\end{tabular}

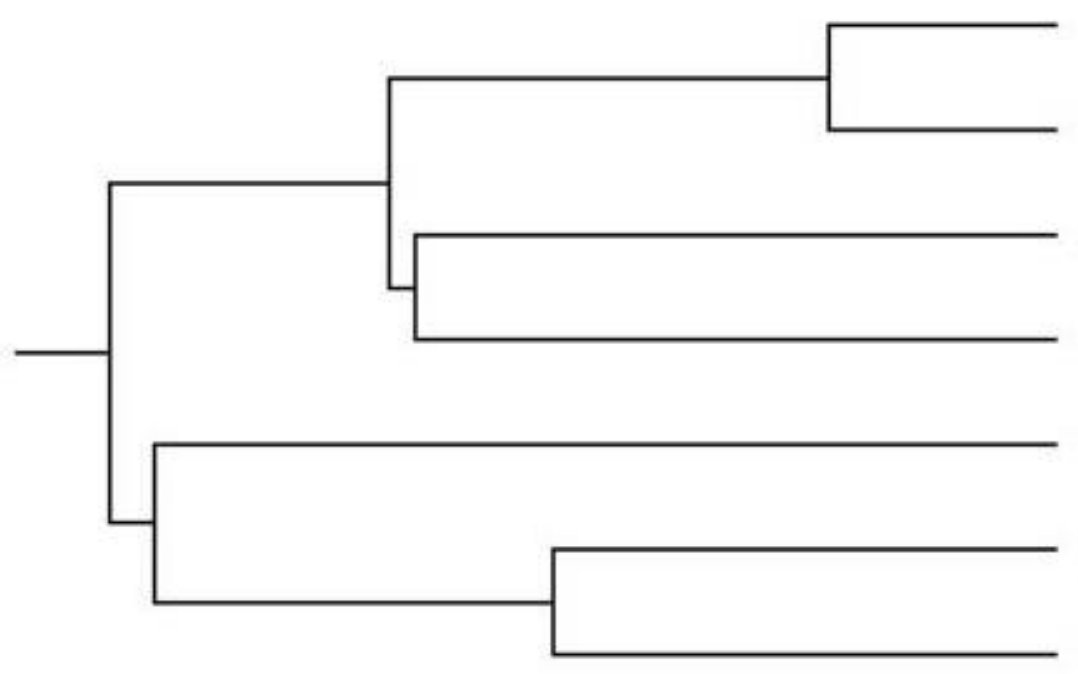

FiguRa 2. Dendrograma de similitud de las estaciones de muestreo en ambas épocas (2002 y 2003), utilizando las capturas por unidad de esfuerzo transformadas $(\log x+1)$, el índice de Bray Curtis y el método de la media ponderada.

FIGURE 2. Dendrogram of similarity of the sampling stations in both seasons (2002 and 2003), using the capture per effort unit transformed with $\log (\mathrm{x}+1)$, the Bray Curtis index and the group average method. 
Ictiofauna del río Quilque: НАBIT, E. ET AL.

TABLA V. Promedio y desviación estándar de la longitud total ( $\mathrm{mm}$ ), peso (g) y factor de condición promedio de las especies encontradas en el río Quilque en ambos muestreos (2002: sin planta de tratamiento, 2003: con planta de tratamiento).

TABLE V. Mean and standard deviation of the total length $(\mathrm{mm})$, weight $(\mathrm{g})$ and condition factor of the different species found in the Quilque stream in both sampling seasons (2002: without treatment plant, 2003: with treatment plant).

\begin{tabular}{lccccc}
\hline Especie & $\begin{array}{c}\text { Época } \\
\text { de muestreo }\end{array}$ & $\begin{array}{c}\text { Estación } \\
\text { de muestreo }\end{array}$ & $\begin{array}{c}\text { Longitud } \\
\text { Total }(\mathbf{m m})\end{array}$ & Peso (g) & $\begin{array}{c}\text { Factor } \\
\text { de condición }\end{array}$ \\
\hline B. australis & 2002 & E1 & $208.6 \pm 147.4$ & $186.3 \pm 234.5$ & $0.88 \pm 0.24$ \\
& & E2 & $50.5 \pm 0.00$ & $0.9 \pm 0.00$ & $0.70 \pm 0.00$ \\
C. galusdae & 2002 & E1 & $45.6 \pm 4.46$ & $1.19 \pm 0.35$ & $1.24 \pm 0.13$ \\
& & E2 & $49.1 \pm 0.00$ & $1.56 \pm 0.00$ & $1.31 \pm 0.00$ \\
& 2003 & E2 & $53.4 \pm 4.74$ & $1.26 \pm 0.32$ & $1.04 \pm 0.13$ \\
P. irwini & 2002 & E1 & $71.9 \pm 0.00$ & $4.64 \pm 0.00$ & $1.24 \pm 0.00$ \\
& 2003 & E1 & $5.9 \pm 1.27$ & $1.93 \pm 1.22$ & $0.97 \pm 0.09$ \\
& & E2 & $6.0 \pm 0.36$ & $2.5 \pm 0.45$ & $1.17 \pm 0.04$ \\
& & E5 & $3.2 \pm 1.1$ & $0.35 \pm 0.33$ & $0.88 \pm 0.09$ \\
D. nahuelbutaensis & 2003 & E2 & $46.8 \pm 0.00$ & $0.94 \pm 0.00$ & $0.92 \pm 0.00$ \\
T. areolatus & 2003 & E1 & $84.3 \pm 15.69$ & $3.9 \pm 2.51$ & $0.58 \pm 0.08$ \\
& & E2 & $112.4 \pm 30.33$ & $10.7 \pm 9.10$ & $0.64 \pm 0.06$ \\
& & E3 & $50.7 \pm 21.17$ & $1.13 \pm 6.8$ & $0.70 \pm 0.07$ \\
& & E4 & $43.4 \pm 7.89$ & $0.60 \pm 0.27$ & $0.71 \pm 0.05$ \\
B. bulloki & 2003 & E1 & $35.5 \pm 47.96$ & $0.28 \pm 0.12$ & $1.05 \pm 0.54$ \\
O. mykiss & 2003 & E1 & $265.0 \pm 0.00$ & $199.1 \pm 0.00$ & $1.07 \pm 0.00$ \\
\hline
\end{tabular}

\section{DISCUSION}

El río Quilque presenta un enriquecimiento de sus aguas, debido al continuo aporte de los residuos domésticos de la ciudad de Los Angeles y de otras fuentes ubicadas aguas arriba, principalmente de origen agrícola. Al comparar la calidad del agua luego de la puesta en marcha de la planta de tratamiento, se observa una leve mejoría, principalmente en las variables $\mathrm{DBO}_{5}$, sólidos suspendidos totales y nitrógeno. En el caso de los coliformes fecales, la mejoría se manifiesta en menor grado, dado que el río Quilque conduce una contaminación fecal importante desde aguas arriba. Además, persisten remanentes de contaminantes en los sedimentos del lecho de río, que hasta el momento del estudio no han sido degradados o transportados aguas abajo.

Las alteraciones que ha experimentado este ecosistema, ya sea en su calidad del agua como en su estructura, han provocado el empobrecimiento de la ictiofauna, tanto en su riqueza de especies como en su abundancia, lo cual es característico de efectos de largo plazo (Wolter et al. 2000). Las únicas especies encontradas en el primer muestreo son propias de ambientes con velocidades de corriente moderadas a bajas, presencia de vegetación acuática y contenidos medios de materia orgánica (Campos et al. 1993, Ruiz 1993, Habit et al. 2003), por lo que se encontraron presentes sólo fuera del área de influencia de las descargas domésticas. El resto del curso estudiado se encontraba despoblado de fauna íctica, al igual que las estaciones E6 a la E8 en la situación con planta de tratamiento. La ausencia de peces en estos sectores queda explicado por la alta carga de $\mathrm{DBO}_{5}$, la cual continúa con concentraciones cercanas o sobre el límite tolerado por los peces (10 $\mathrm{mg} / \mathrm{L} \mathrm{DBO}_{5}$, Gafny et al. 2000). Esta situación ha sido descrita para otros ríos que reciben efluentes domésticos sin tratar, en los que tanto los peces como los crustáceos quedan restringidos sólo a los tramos con bajas concentraciones de $\mathrm{DBO}_{5}$ (Dyer et al. 2003).

El incremento en el número de especies y distribución espacial de la ictiofauna en el área evidencia una 
leve recuperación entre las situaciones sin y con planta de tratamiento de aguas servidas. La presencia de especies como D. nahuelbutaensis y Oncorhynchus mykiss, características de aguas de buena calidad, así como la mayor abundancia de $T$. areolatus y $P$. irwini, evidencian un proceso de recolonización de los tramos del río ubicados entre las estaciones E1 y E5. Además, la presencia de las especies nativas $T$. areolatus y $P$. irwini, en los tramos del río que previamente se encontraron sin peces, manifiesta su capacidad colonizadora de ambientes alterados, lo cual ya había sido reportado en canales de riego y ríos sometidos a modificaciones de su cauce (Habit \& Parra 2001, Habit et al. 2002).

Al contrario de lo encontrado en otros sistemas alterados por descargas de aguas servidas (Gafny et al. 2000, Dyer et al. 2003) y en sistemas lénticos eutróficos de Chile (Parra et al. 2003), la ictiofauna del Quilque está compuesta mayoritariamente por especies nativas. Parra et al. (2003) reportaron la dominancia de especies introducidas tolerantes como la carpa común (Cyprinus carpio Linneo, 1758) y pejerreyes argentinos (Odontesthes bonariensis (Valenciennes, 1835) en lagos de alta trofía. La falta de tales especies en el río Quilque puede ser explicada por la ausencia de ambientes de mayor profundidad, necesarios para su desarrollo. Al contrario, en el río Quilque predominan los ambientes someros (ca. 0,6 m), propicios para especies como T. areolatus y P. irwini.

Dado que los procesos de recuperación de los ecosistemas fluviales son lentos (González del Tánago \& García de Jalón 1998), es esperable que a largo plazo se restablezca una comunidad de peces más diversa y abundante. Para que ello ocurra es necesario no sólo reducir las descargas de aguas servidas, sino también abatir los ingresos de materia orgánica que conduce el río desde aguas arriba de la ciudad de Los Angeles.

\section{AGRADECIMIENTOS}

Agradecemos a Waldo San Martín y Miguel Vilches por su colaboración en la toma de muestras. Este trabajo fue parcialmente financiado por los proyectos DIUC 203.113.064-1.0. y 204.310.041-1.0.

\section{BIBLIOGRAFIA}

Alarcón, E. \& R. Román. 2003. Análisis del aporte de carga orgánica en nuevas plantas de tratamiento de aguas servidas de Rancagua, Los Angeles y Curicó. Resúmenes XV Congreso de Ingeniería Sanitaria y Ambiental AIDIS - Chile, 1-14 pp.

ApHA. 1998. Standard Methods for the Examination of Water and Wastewater. American Public Health Association, the American Water Works Association and the Water Environment Federation. 20th Edition. $1220 \mathrm{pp}$.

Bray, J.R. \& J.T. CURTIS. 1957. An ordination of the upland forest communities of southern Wisconsin. Ecol. Monogr., 27:325-349.

Campos, H., V.H. Ruiz, J.F. Gavilán \& F. Alay. 1993. Peces del Río Biobío. Serie: Publicaciones de Divulgación EULA, Editorial Universidad de Concepción, Concepción, 5: 100 p.

Campos, H., G. Dazarola, B. Dyer, L. Fuentes, J.F. Gavilán, L. Huaquín, G. Martínez, R. Meléndez, G. Pequeño, F. Ponce, V.H. Ruz, W. Siefeld, D. Soto, R. Vega \& I. VIla. 1998. Categorías de Conservación de peces nativos de aguas continentales de Chile. Boletín del Museo Nacional de Historia Natural (Chile), 47:101-122.

Colby, P., G.R. Spangler, D.A. Hurley \& A.M. Mccombie. 1972. Effects of eutrophication on salmonid communities in oligotrophic lakes. Journal Fisheries Research Board Canada, 29: 975-983.

Conama. 2002. Instructivo Presidencial para la Dictación de Normas Secundarias de Calidad Ambiental para Aguas Continentales Superficiales y Aguas Marinas. Acuerdo No 210/02, Consejo Directivo de CONAMA.

Dyer, S., C. Peng, D. Mcavoy, N. Fendinger, P. MassCheleyn, L. Castillo \& J.M. Lim. 2003. The influence of untreated wastewater to aquatic communities in the Balatuin River, The Philippines. Chemosphere, 52(1):43-53.

Field, J.G., K.R. Clarke \& R.M. Warwick. 1982. A practical strategy for analysing multispecies distribution patterns. Mar. Ecol. Prog. Ser., 8: 37-52.

Gafny, S., M. Goren \& A. Gasith. 2000. Habitat condition and fish structure in a coastal mediterranean stream (Yarqon, Israel) receiving domestic effluent. Hidrobiología, 422/423: 319-330.

Ganasan, V. \& R.M. Hughes. 1998. Application of an index of biological integrity (IBI) to fish assemblages of the rivers Khan and Kshipra (Madhya Pradesh), India. Freshwater Biology 40(2):367-383.

González del Tánago, M. \& D. García de Jalón. 1998. Restauración de Ríos y Riberas. Escuela Técnica Superior de Ingenieros de Montes. Ediciones Mundi Prensa, $317 \mathrm{pp}$.

HABIT, E. \& O. PARRA. 2001. Impactos ambientales de los canales de riego sobre la fauna de peces. Ambiente y Desarrollo, 27(3): 50-56.

Habit, E., O. Parra, C. Valdovinos, S. Basualto, G 
Azócar \& P. Victoriano. 2002. Programa de Seguimiento Ambiental de la Central Hidroeléctrica Rucúe. Informe Final Ejecutivo. Oficina de Asistencia Técnica, Centro EULA-Chile, Universidad de Concepción, 50 pp.

Habit, E., P. Victoriano \& A. Rodríguez-Ruíz. 2003. Variaciones espacio-temporales del ensamble de peces de un sistema fluvial de bajo orden del centro- sur de Chile. Revista Chilena de Historia Natural. 76: 3-14.

MinseCPRE. 2001. Decreto $\mathrm{N}^{\circ} 90$. Norma de emisión para la regulación de contaminantes asociados a las descargas de residuos líquidos a aguas marinas y continentales superficiales. Publicado en el Diario Oficial de la República de Chile el 7 de Marzo.

Magurran, A.E. 1988. Ecological Diversity and its measurement. Croom Helm. London. 88 pp.

Parra, O., C. Valdovinos, R. Urrutia, M. Cisternas, E. Habit \& M. Mardones. 2003. Caracterización y tendencias tróficas de cinco lagos costeros de Chile central. Limnetica, 22(1-2): 51-83.

Porter, C.M. \& D.M. Janz. 2002. Treated municipal sewage discharge affects multiple levels of biological organization in fish. Ecotoxicology and Environmental Safety. 54: 199-206.

RuIz, V.H. 1993. Ictiofauna del río Andalién (Concepción, Chile). Gayana Zoología (Chile), 57: 109 - 278.

Saunders, D.L., J.J. Meeuwig \& A.C. Vincent. 2002. Freshwater Protected Areas: Strategies for Conservation. Conservation Biology, 16(1): 3041.

SCrimgeour, G. \& P. Chambers. 2000. Cumulative effects of pulp mill and municipal effluents on epilithic biomass and nutrient limitation in a large northern river ecosystem. Canadian Journal Fisheries Aquatic Science, 57: 1342-1354.

VOLLENWEIDER, R.A. 1968. Scientifical fundamentals of the eutrophication of lakes and flowing waters, with particular reference to nitrogen and phosphorus as factors in eutrophication. Rep. Organis. Econ. Coop. And Dev., DAS/CSI/68.27. Paris.

Wolter, C., J. Minow, A.Vilcinskas \& U. Grosch. 2000. Long-term effects of human influence on fish community structure and fisheries in Berlin waters: an urban water system. Fisheries Management \& Ecology, 7(1): 97-104. 